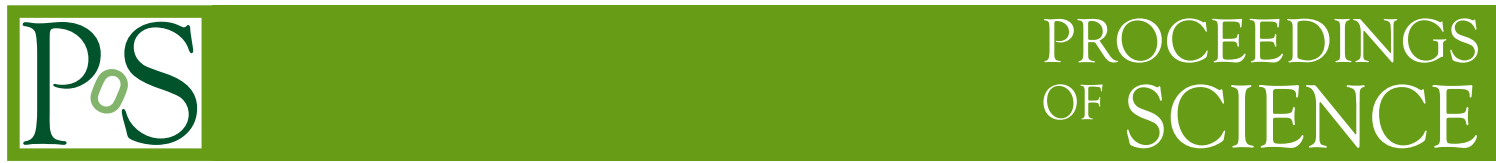

\title{
Pion physics in the strong coupling limit: A poor man's QCD?
}

\section{J. Cecile*}

Department of Physics, Duke University, Durham, NC 27708, USA

E-mail: djcecile@phy.duke.edu

To model pions of two-flavor QCD we construct a lattice field theory involving two flavors of staggered quarks interacting strongly with $U(1)$ gauge fields. In the massless quark limit, this theory has an $S U_{L}(2) \times S U_{R}(2) \times U_{A}(1)$ symmetry. By introducing a four-fermion term we can break the $U_{A}(1)$ symmetry and thus incorporate the physics of the QCD anomaly. We can also tune the pion decay constant $F$, to be small compared to the lattice cutoff by starting with an extra fictitious dimension, thus allowing us to model low energy pion physics in a setting similar to lattice QCD from first principles. However, unlike lattice QCD, a major advantage of our model is that we can easily design efficient algorithms (Directed Path Algorithms) to compute a variety of quantities in the chiral limit. Here we show that the model is consistent with the predictions of chiral perturbation theory in the $\varepsilon$-regime.

The XXV International Symposium on Lattice Field Theory

July 30 - August 42007

Regensburg, Germany

\footnotetext{
${ }^{*}$ Speaker.
} 


\section{Introduction}

Current lattice calculations are typically carried out at unphysically large quark masses, and chiral perturbation theory is used to extrapolate to realistic quark masses. A study of the range of quark masses over which the chiral expansion may be safely applied is thus useful.

One way to check the reliability of chiral extrapolations is to verify them in different regimes. There are many such regimes depending on the values of the pion masses and the physical box sizes, but the most popular examples are the $p$-regime and the $\varepsilon$-regime. Interestingly, in all the regimes the extrapolation formulas depend on the same low energy constants that describe the chiral Lagrangian. Hence if the data from a lattice calculation can be fit in both the regimes with the same low energy constants one would gain more confidence in the extrapolations.

Studies of QCD-like models may teach us more about chiral extrapolations. Motivated by this, we study a lattice field theory model of pions in two-flavor QCD which we previously introduced $[1,2,3,4]$. This model is particularly attractive since it is very much like QCD in its formulation but we are easily able to design very efficient algorithms to study a variety of quark masses.

\section{Model, Algorithm, and Observables}

The Euclidean space action of our model is (note that the usual factor of $\frac{1}{2}$ in the fermion kinetic term has been absorbed into the fields):

$$
S=-\sum_{x} \sum_{\mu=1}^{d+1} \eta_{\mu, x}\left[\mathrm{e}^{i \phi_{\mu, x}} \bar{\psi}_{x} \psi_{x+\hat{\mu}}-\mathrm{e}^{-i \phi_{\mu, x}} \bar{\psi}_{x+\hat{\mu}} \psi_{x}\right]-\sum_{x}\left[m \bar{\psi}_{x} \psi_{x}+\frac{\tilde{c}}{2}\left(\bar{\psi}_{x} \psi_{x}\right)^{2}\right]
$$

where $x$ denotes a lattice site on a $d+1$ dimensional hyper-cubic lattice $L_{t} \times L^{d}$. $L_{t}$ is a fictitious time direction and will be used to tune $F$, the non-perturbative physical mass scale, to be small compared the lattice cutoff. $L^{d}$ is the usual Euclidean space-time box. $\bar{\psi}_{x}$ and $\psi_{x}$ are two component Grassmann fields that represent the two quark $(u, d)$ flavors of mass $m$, and $\phi_{\mu, x}$ is the compact $U(1)$ gauge field through which the quarks interact. $\mu=1,2, \ldots, d, d+1$ runs over the $d+1$ directions and the $\mu=1$ direction denotes the fictitious temperature, while the remaining directions represent Euclidean space-time. The staggered fermion phase factors $\eta_{\mu, x}$ obey: $\eta_{1, x}^{2}=T$ and $\eta_{i, x}^{2}=1$ for $i=2,3, \ldots, d+1$. $T$ controls the fictitious temperature and will be used to tune to the continuum limit $(F \ll 1)$. The coupling $\tilde{c}$ will set the strength of the anomaly.

When $\tilde{c}, m=0$, the action exhibits a global $S U_{L}(2) \times S U_{R}(2) \times U_{A}(1)$ symmetry like $N_{f}=2$ QCD. The partition function of this model is equivalent to that of a classical statistical mechanics model involving configurations made up of gauge invariant objects, namely monomers, dimers, pion loops, and instantons [5]. These configurations and the related constraints are discussed in more detail in [4].

To study this model, we have designed a Directed Loop Algorithm [6]. The algorithm and the many observables such as two point correlation functions, susceptibilities, and current susceptibilities, are discussed in [4]. 


\section{Chiral Perturbation theory in the $\varepsilon$-regime}

In the phase of broken chiral symmetry and large anomaly, the low energy physics of our model is described by the Euclidean chiral Lagrangian

$$
\mathscr{L}=\frac{F^{2}}{4} \operatorname{tr}\left(\partial_{\mu} U^{\dagger} \partial_{\mu} U\right)-m \Sigma \operatorname{tr}\left(U+U^{\dagger}\right)
$$

where $F$ is the chiral pion decay constant, $\Sigma$ is the chiral condensate, and $U \in S U(2)$ is the pion field. The $\varepsilon$-regime is the limit where $L$, the linear size of the four dimensional hypercube, is large such that $F L \gg 1$ but $m \Sigma L^{4}$ is fixed. Many observables in the $\varepsilon$-regime have been computed and can be found in the literature. For example the behavior of the condensate susceptibility $\chi_{\sigma}$ as a function of $L$ at $m=0$ was obtained in [7] for the $O(N)$ model. The $N=4$ result is

$$
\chi_{\sigma}=\frac{\Sigma^{2} L^{4}}{4}\left[1+\frac{3 \beta_{1}}{(F L)^{2}}+\frac{a}{(F L)^{4}}+\ldots\right]
$$

Further, the chiral and vector current susceptibilities $Y_{c}$ and $Y_{v}$ (respectively) were obtained in [8]

$$
\begin{aligned}
& Y_{c}=\frac{F^{2}}{2}\left(\left\{1+\frac{\beta_{1}}{(F L)^{2}}+\frac{a^{\prime}}{(F L)^{4}}+\ldots\right\}+\frac{u^{2}}{24}\left\{1+\frac{3 \beta_{1}}{(F L)^{2}}+\frac{b_{c}}{(F L)^{4}}+\ldots\right\}+\mathscr{O}\left(u^{4}\right)\right) \\
& Y_{v}=\frac{F^{2}}{2}\left(\left\{1+\frac{\beta_{1}}{(F L)^{2}}+\frac{a^{\prime}}{(F L)^{4}}+\ldots\right\}-\frac{u^{2}}{24}\left\{1+\frac{3 \beta_{1}}{(F L)^{2}}+\frac{b_{v}}{(F L)^{4}}+\ldots\right\}+\mathscr{O}\left(u^{4}\right)\right)
\end{aligned}
$$

for small $u=\Sigma m L^{4}\left[1+3 \beta_{1} /\left(2(F L)^{2}\right)\right]$. In these relations $\beta_{1}=0.14046$ is the shape coefficient and $a, a^{\prime} b_{c}, b_{v}$ are constants that depend on higher order low energy constants. Also $Y_{c}=Y_{v}$ at $u=0$ reflects the chiral symmetry of the theory.

We show that the calculations in our model are consistent with Eqs.(3.2) and (3.3). We choose $c=0.3$ and $T=1.733$ with fixed $L_{t}=2$. These parameters are chosen to ensure that chiral symmetry is spontaneously broken and the anomalous pion mass $\left(M_{\eta}\right)$ is about 1 in lattice units. At this $T, F \sim 0.1$ in lattice units, which should make our results less sensitive to lattice artifacts. Fig.(1) shows data for $Y_{c}$ as a function of $L$ for $m=0$. The solid lines are fits to Eq.(3.3). The fits are very good if we allow $a^{\prime} \neq 0$ and fit to lattice sizes above $L \sim 24$. We can then extract $F=0.0992(1)$ and $a^{\prime}=2.7(1)$ with a $\chi^{2} / D O F=0.8$. However, as shown in the figure, this means that setting $a^{\prime}=0$, i.e.. using only the leading correction in the chiral expansion, will not be a good approximation for $L<48$. This is a result of the smallness of $a^{\prime} / \beta_{1}$. In other words, although our data is consistent with the Eq.(3.3), unfortunately we are not sensitive to the universal corrections at order $\mathscr{O}\left(1 / L^{2}\right)$.

We now consider the condensate susceptibility $\chi_{\sigma}$. Fig(2) is a plot $\chi_{\sigma} / L^{4}$ as a function of $L$. The solid line is a fit to the data using the Eq.(3.2) where $F=0.0992$ is fixed and only data for $L>20$ is used in the fit. We find $\Sigma=0.1862(2), a=3.0(2)$ with a $\chi^{2} / D O F=1.3$. Again, the universal correction at order $\mathscr{O}\left(1 / L^{2}\right)$ is small compared to the next order non-universal correction for $L<48$. 


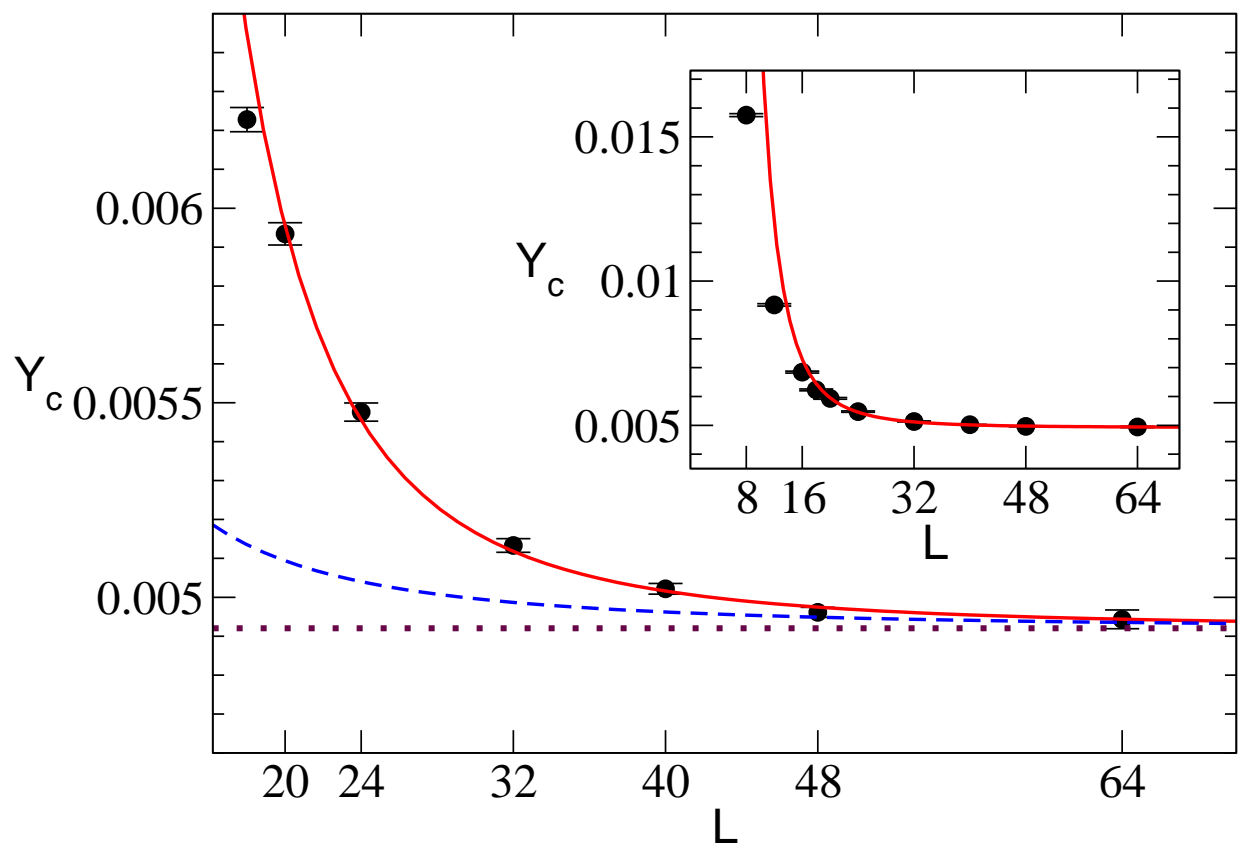

Figure 1: Vector current susceptibility $Y_{C}$ as a function of $L$ at $T=1.733, c=0.3$ and $m=0\left[Y_{C}=Y_{V}\right]$. The solid line shows the fit with $F=0.0992$ and $a^{\prime}=2.7$. The dashed line shows the same curve but with $a^{\prime}=0$, and the dotted line shows the infinite volume limit $F^{2} / 2$.

We have neglected the $\log (L)$ corrections that arise at the order $1 /(F L)^{4}$ [9]. The reason is as follows: Consider the chiral condensate susceptibility in Eq.(3.2). Using the results of [7, 9, 10], we can obtain the logarithmic corrections to Eq. (3.2). In particular it follows that

$$
\chi_{\sigma}=\frac{\Sigma^{2} L^{4}}{4}\left[1+\frac{3 \beta_{1}}{F^{2} L^{2}}+\frac{1}{F^{4} L^{4}}\left\{\alpha+\frac{15}{16 \pi^{2}}(\log F L)\right\}+O\left(\frac{1}{F^{5} L^{5}}\right)\right]
$$

where now $\alpha=\left(3 \beta_{1}^{2}+15 \beta_{2}\right) / 2+3\left[\log \left(\Lambda_{M} / F\right)+4 \log \left(\Lambda_{\Sigma} / F\right)\right] / 16 \pi^{2}$ and $\beta_{2}=-0.020305$ is another shape coefficient. The mass scales $\Lambda_{M}, \Lambda_{\Sigma}$ represent the non-universal information of the model and are defined in [7]. Assuming $L_{1}=20$ is the smallest lattice size and $L_{2}=64$ is the largest lattice size we use in the fits, the change in the logarithmic correction term $15 \log \left(L_{2} / L_{1}\right) /\left(16 \pi^{2}\right) \sim$ 0.1 is within errors of the constant $a=3.0$ (2) obtained above by fitting the $\chi_{\pi}$ data to Eq.(3.2). Thus, our errors are still large and we are not yet sensitive to the logarithmic corrections. Interestingly, since $15 \log (F L) /\left(16 \pi^{2}\right)$ is much smaller than $a$ in the region of our fits, we estimate that $a \sim 3\left[\log \left(\Lambda_{M} / F\right)+4 \log \left(\Lambda_{\Sigma} / F\right)\right] /\left(16 \pi^{2}\right)$, which means that $\left[\log \left(\Lambda_{M} / F\right)+4 \log \left(\Lambda_{\Sigma} / F\right)\right] \sim 150$ is unnaturally large, and the factor $\frac{1}{16 \pi^{2}}$ is essential to keep the coefficient of $1 /(F L)^{4}$ of order 1 . In other words factors like $\frac{1}{16 \pi^{2}}$ cannot always be assumed to be small since they can be multiplied by large numbers.

Having confirmed that the results are consistent with Eq.(3.3) when $u=0$, we can also check that for consistentency with Eq.(3.3) at order $u^{2}$. A way to do this is to tune the quark mass 


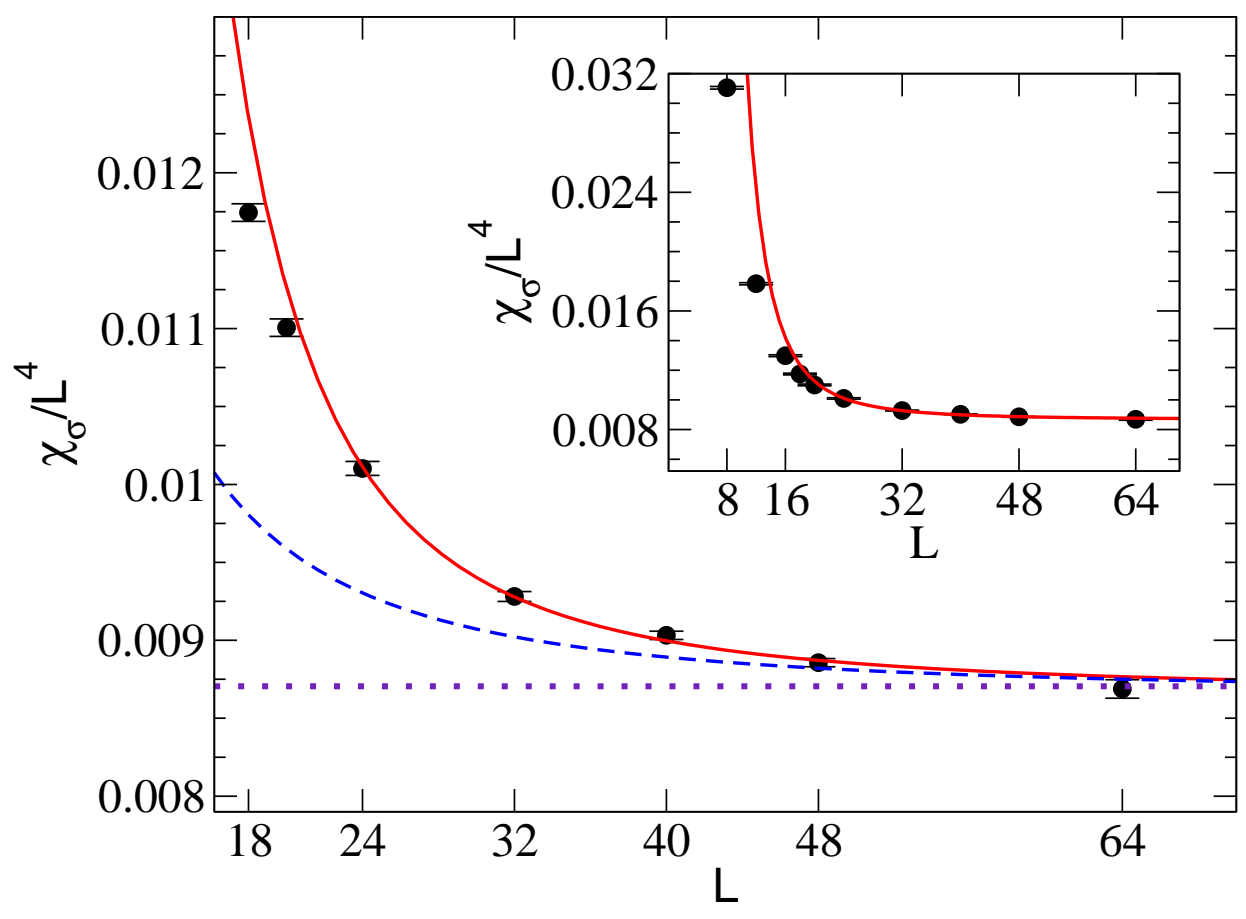

Figure 2: Chiral condensate susceptibility $\chi_{\sigma}$ as a function of $L$ at $T=1.733, c=0.3$, and $m=0$. The solid line shows the fit with $\Sigma=0.1866, F=0.0992$ and $a=3.0$. The dashed line shows the same curve with $a=0$, and the dotted line shows the infinite volume limit $\Sigma^{2} / 4$.

and the volume such that $u$ is fixed and small but this is tedious. For example even at $u=1$ for $L=48$ the quark mass should be as small as $10^{-6}$. Since $m^{2}$ is involved in a probability, double precision arithmetic may become an issue. Thus, here we devise another method. To understand our approach we expand the partition function in powers of the quark mass

$$
Z=Z_{0}+m^{2} Z_{2}+m^{4} Z_{4}+\ldots
$$

where $Z_{n}$ is obtained from configurations with $n$ monomers. In this expansion we neglect the $m^{2}$ contribution to instanton weights as they will not contribute in the $\varepsilon$ regime. Similarly, let $Y_{v}^{(n)}$ and $Y_{c}^{(n)}$ denote the values of the current susceptibilities when computed in the $n$ monomer sector. Expanding observables in the various monomer sectors it follows that

$$
Y_{i}=Y_{i}^{(0)}+m^{2}\left\{Y_{i}^{(2)}-Y_{i}^{(0)}\right\} \frac{Z_{2}}{Z_{0}}+\ldots
$$


where $i=v, c$. Following the analysis in [4], we conclude that

$$
\begin{aligned}
& Y_{c}^{(2)}=\frac{2 F^{2}}{3}\left(1+\frac{3 \beta_{1}}{2(F L)^{2}}+\frac{b_{c}^{\prime}}{(F L)^{4}}+. .\right) \\
& Y_{v}^{(2)}=\frac{F^{2}}{3}\left(1+\frac{b_{v}^{\prime}}{(F L)^{4}}+. .\right)
\end{aligned}
$$

Notice that $Y_{c}^{(2)} \neq Y_{v}^{(2)}$ because of explicit chiral symmetry breaking that is introduced by the presence of the monomers.

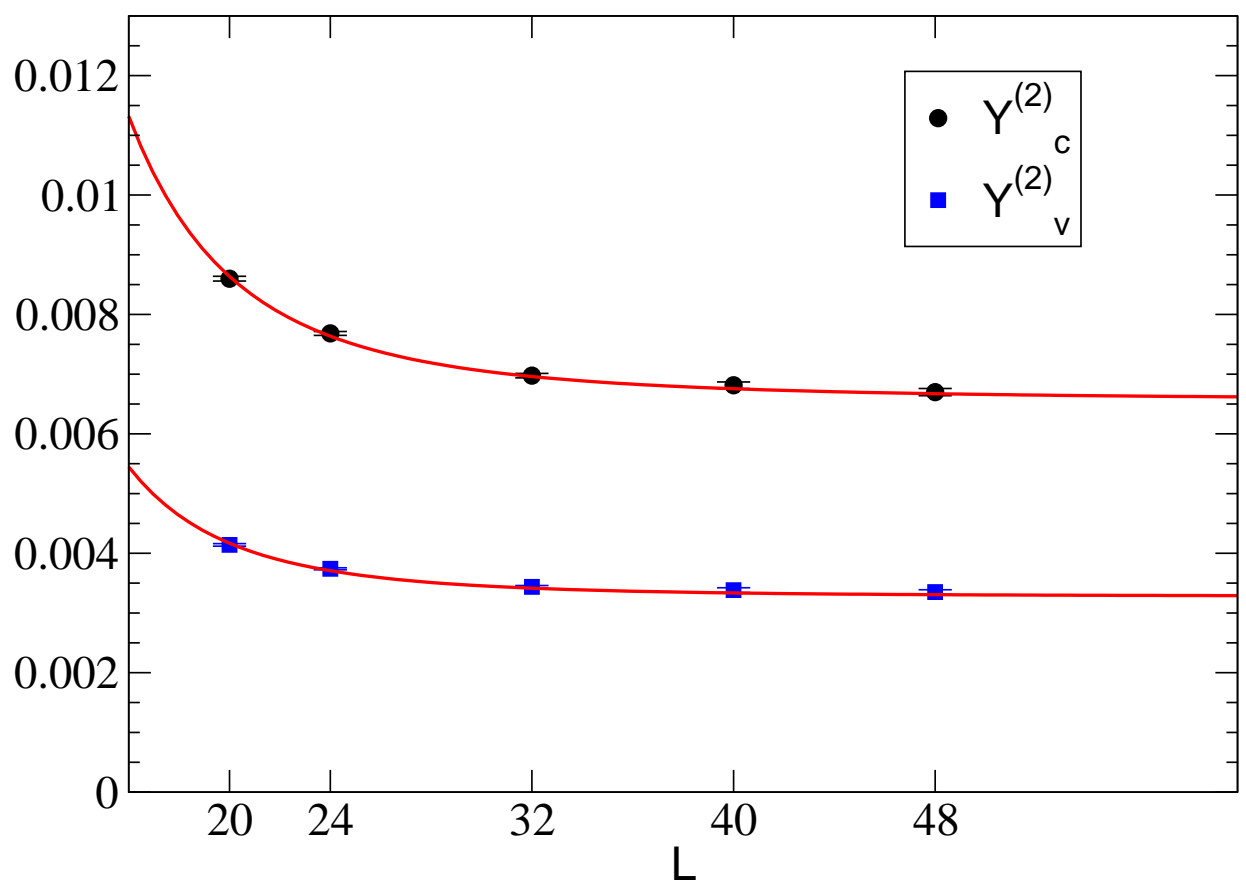

Figure 3: Plot of $Y_{c}^{(2)}$ and $Y_{v}^{(2)}$, evaluated in the two monomer sector as a function of $L$ at $T=1.733$, $c=0.3$ and $m=0$. The solid lines are fits as discussed in the text.

We used the fixed monomer update to compute $Y_{c}^{(2)}$ and $Y_{v}^{(2)}$. Fig.(3) shows our results as a function of $L$. We fixed $F=0.0992$ and fit the data to Eq.(3.7). We found $b_{c}^{\prime}=4.1(1)$ with a $\chi^{2} / D O F=1.1$, and $b_{v}^{\prime}=4.2(1)$ with a $\chi^{2} / D O F=2.1$. Although the results again appear consistent with the predictions at large $L$, the large values for the constants $b_{c}^{\prime}$ and $b_{v}^{\prime}$ show that more data with small errors for $L>48$ is needed to be sure we can be sensitive to the universal predictions at $\mathscr{O}\left(1 / L^{2}\right)$.

\section{Conclusions and Future Work}

We have illustrated a new approach to modeling the physics of pions in $N_{f}=2$ QCD. We used $N_{f}=2$ lattice QED in the strong coupling limit and have demonstrated that by using a mapping to 
dimer models we can explore the chiral limit very efficiently. We have shown consistency with the $\varepsilon$-regime predictions of chiral perturbation theory. We have also demonstrated that we can make $F \ll 1$ by tuning a fictitious temperature so one approaches a second order phase transition. This tuning helps remove lattice artifacts and approach a continuum-like theory.

We are currently performing calculations in the $p$-regime and plan to test the predictions of chiral perturbation theory in this regime. We plan to study the range of $m$ from which chiral perturbation theory will allow us to compute $F$ and $\Sigma$ in the $p$-regime and to check if this analysis is consistent with the results we presented here for the $\varepsilon$-regime. Since our algorithm allows us to compute the chiral values directly we have the ability to make direct comparisions and thus check the reliability of chiral extrapolations found in the literature.

\section{Acknowledgments}

This work was done in collaboration with S. Chandrasekharan. It was partially supported by the DOE grant DE-FG02-05ER41368. The author thanks B.C. Tiburzi and F.J. Jiang for useful discussions.

\section{References}

[1] S. Chandrasekharan and A.C. Mehta, PoS LAT2006 128 (2006).

[2] D. J. Cecile PoS LAT2006 207 (2006)

[3] S. Chandrasekharan and A.C. Mehta, Phys. Rev. Lett. 99, 142004 (2007).

[4] D. J. Cecile and S. Chandrasekharan, arXiv:0708.0558

[5] P. Rossi and U. Wolff, Nucl. Phys. B248, 105 (1984).

[6] D.H. Adams and S. Chandrasekharan, Nucl. Phys. B662, 220 (2003).

[7] P. Hasenfratz and H. Leutwyler, Nucl. Phys. B343, 241 (1990).

[8] F. C. Hansen and H. Leutwyler, Nucl. Phys. B350, 201 (1991).

[9] M. Gockeler, K. Jansen, and T. Neuhaus, Phys. Lett. B273, 4501991.

[10] A. Hasenfratz et al., Nucl. Phys. B356, 332 (1991). 